\title{
Kernos
}

Revue internationale et pluridisciplinaire de religion grecque antique

9 | 1996

Varia

\section{Le Cent-Bras Briarée, fils de Zeus plus fort que son} père

Une correction de la Théogonie (886-900) dans l'Iliade (I, 393-406)

\section{Alain Ballabriga}

\section{(2) OpenEdition}

Journals

Édition électronique

URL : http://journals.openedition.org/kernos/1175

DOI : 10.4000/kernos. 1175

ISSN : 2034-7871

Éditeur

Centre international d'étude de la religion grecque antique

Édition imprimée

Date de publication : 1 janvier 1996

ISSN : 0776-3824

Référence électronique

Alain Ballabriga, "Le Cent-Bras Briarée, fils de Zeus plus fort que son père », Kernos [En ligne], 9 | 1996, mis en ligne le 21 avril 2011, consulté le 01 mai 2019. URL : http://journals.openedition.org/

kernos/1175; DOI : 10.4000/kernos.1175 


\section{Le Cent-Bras Briarée, fills de Zeus plus fort que son père}

Une correction de la Théogonie (886-900) dans l'Iliade (I, 393-406)

Le chant I de l'Iliade commence par exposer les raisons de la «colère (mênis) d'Achille ", véritable moteur du poème puisqu'il narre pour l'essentiel l'avantage momentané des Troyens et les revers des Grecs consécutifs au retrait volontaire de leur principal champion, en proie à son courroux. À la suite d'une peste envoyée sur l'armée achéenne par le dieu Apollon, Agamemnon doit rendre la captive qui lui était échue, Chryséis, à son père Chrysès, prêtre d'Apollon. Pour se dédommager, Agamemnon fait enlever à Achille sa captive et concubine Briséis. Ulcéré, Achille implore, au bord de la mer, sa mère, la Néréide Thétis, qui sort du fond des flots où elle réside auprès du Vieux de la Mer, son père Nérée. Après lui avoir exposé les événements qui motivent son courroux, Achille s'exprime en ces termes :

À toi donc, si tu peux, de venir en aide à ton vaillant fils. Va vers l'Olympe et supplie Zeus, si aussi bien tu as jadis, par parole ou par acte, servi ses désirs. Dans le palais de mon père, souvent je t'ai ouïe t'en glorifier. Tu disais comment, seule entre les immortels, tu avais du Cronide à la nuée noire écarté

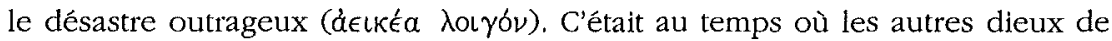
l'Olympe prétendaient l'enchaîner ( $\xi v v \delta \tilde{\sigma} \sigma a \mathrm{l})$ - Héré et Poséidon et Pallas Athéné. Mais toi, tu vins à lui; tu sus, toi, déesse, le soustraire à ces chaînes

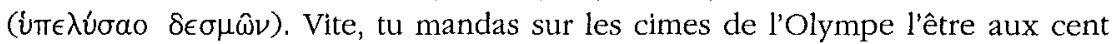

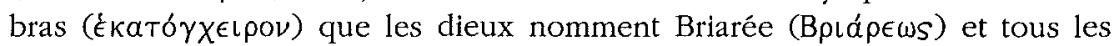
hommes Égéon (Ai $\gamma a i(\omega)$ ), et qui, pour la force, surpasse son père même (ó

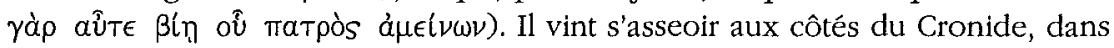
l'orgueil de sa gloire. Les bienheureux, à sa vue, prirent peur et n'enchaînèrent

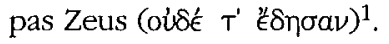

Après quoi Achille suggère à sa mère d'obtenir que Zeus aide les Troyens à repousser à la mer les Achéens décimés. Thétis va donc trouver Zeus, assis à l'écart sur le plus haut sommet de l'Olympe, et le supplie de donner l'avantage aux Troyens pour que les Achéens honorent Achille. Le maitre des cieux donne alors un signe d'assentiment qui fait trembler l'Olympe (v. 530). L'action de l'Iliade se trouve ainsi lancée.

1 Iliade, I, 393-406. 
Avant d'en revenir aux paroles d'Achille que je viens de citer et qui constituent l'objet de cet article, il est bon de rappeler que la scène olympienne qui occupe la fin du chant 1 présente du pouvoir de Zeus et de ses relations avec les Olympiens une vision très différente de ce que paraît impliquer la requête d'Achille à Thétis. D'abord cette dernière se montre beaucoup moins explicite que son fils sur la nature du service qu'elle a pu jadis rendre à Zeus. Ensuite tous les dieux témoignent à l'égard de Zeus de la plus grande déférence et soumission. On est loin du temps des rébellions! Quant à Héra elle-même, elle est opposée au projet de Zeus, Thétis et Achille mais ne peut rien contre la toute-puissance de son époux. Comme le rappelle Héphaiistos, Zeus pourrait précipiter Héra à bas de son siège « car il est de beaucoup le plus fort » (ó

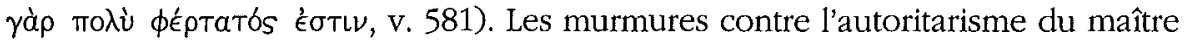
de l'Olympe disparaissent alors pour faire place au plaisir du festin des dieux. Cette vision de l'Olympe est constante dans l'Iliade: Zeus y est un maître sinon incontesté du moins assez puissant pour que ni son épouse ni son frère Poséidon ne songent sérieusement à en découdre avec lui.

Par contre les paroles d'Achille à Thétis semblent dès l'abord impliquer qu'il n'en a pas toujours été ainsi. Il fut un temps où Zeus fut sur le point d'être enchaîné par Héra, Poséidon, ses opposants habituels, mais aussi par Athéna, sa propre fille chérie! Et il ne dut son salut qu'à l'intervention d'un Cent-Bras, Briarée/Égéon, mandé sur l'Olympe par Thétis. Ce curieux épisode, qui contraste donc avec la figure ordinaire de Zeus dans l'Olympe épique, a embarrassé les commentateurs de l'Antiquité jusqu'à nos jours. Qui est ce Briarée/Égéon? À quoi renvoie exactement l'expression «supérieur en force à son père »?

Une première possibilité, exploitée par le philologue Zénodote d'Éphèse, premier directeur de la bibliothèque d'Alexandrie $(v, 285-270 \mathrm{av} \text {. })^{2}$, consistait à athétiser les vers 396-406 («Dans le palais de mon père... et ils n'enchaînèrent pas Zeus. ») ou à récrire le vers le plus problématique pour lui faire dire que Briarée était « pour la force très supérieur à tous ceux qui habitent le Tartare moisi $»^{3}$.

Une autre façon d'accommoder les passages choquants pour la piété et la majesté divine consistait à en proposer une lecture allégorique. En l'occurrence ces lectures furent diverses dans le détail mais dans l'ensemble les allégoristes virent dans le mythe de Briarée aidant Zeus un drame physique et cosmologique du chaud menacé par le froid. Dans cette optique Briarée pouvait être assimilé au soleil ou à Apollon. Comme le fait justement remarquer Félix

2 Sur Zénodote voir l'esquisse récente de Jean LALLOT, Zénodote ou l'art d'accommoder Homère, in Alexandrie IIIe siècle av. J.-C. Tous les savoirs du monde ou le rêve d'universalité des Ptolémées, Dirigé par Chr. JACOB et Fr. DE POLIGNAC, Editions Autrement, 1992 (Série Mémoires, 19), p. 93-99.

3 Voir par exemple l'apparat critique de l'édition de Paul MAZON aux Belles Lettres. 
Buffière, « et dans la circonstance, le fils ne se montre-t-il pas supérieur au père dont il rétablit la souveraineté chancelante ? $\rangle^{4}$

En effet, si aberrantes que puissent à juste titre paraître au lecteur moderne les lectures allégoriques de l'Antiquité, elles ont au moins ici le mérite de nous orienter vers le sens littéral le plus probable de l'expression «supérieur en force à son père », le père de Briarée étant Zeus, à la différence d'une autre interprétation avancée dans les scholies à notre passage, et favorisée par les modernes, à savoir que Briarée avait pour père Poséidon. Cette solution est peu satisfaisante. Cette généalogie n'est nulle part attestée en dehors de nos scholies. Et surtout pourquoi un fils de Poséidon viendrait-il à l'aide de Zeus?

Rappelant les données de la philologie ancienne (à l'exception notable toutefois de l'indication que Félix Buffière avait su extraire des allégoristes) dans le grand «Commentaire de Cambridge » à l'Iliade, Geoffrey Kirk, voici une dizaine d'années, avouait en fin de compte que dans notre passage «bien des choses restent obscures 》 (much remains obscure) ${ }^{5}$.

Mais il semble qu'une voie prometteuse ait été enfin ouverte récemment par le livre de Laura Slatkin sur la figure de Thétis dans l'Iliade ${ }^{6}$. Selon cet auteur, il faut lier l'idée d'un Briarée plus fort que son père à la variante du mythe de Thétis attestée dans la VIIIe Isthmique de Pindare et dans le Prométhée enchaîné d'Eschyle ${ }^{7}$.

L'ode de Pindare rappelle que Poséidon et Zeus durent renoncer à s'unir à Thétis lorsque Thémis eut révélé aux Immortels que la Néréide mettrait au monde un fils qui deviendrait un chef plus puissant que son père ( $\phi \dot{\rho} \rho T \in \rho \circ \nu$

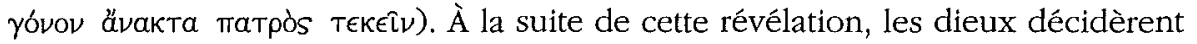
d'octroyer la déesse marine au héros Pélée, le plus pieux des hommes. De cette union entre une déesse et un mortel naquit Achille, le "meilleur des Achéens » dans l'lliade, mortel inférieur aux dieux mais supérieur à tous les autres mortels ${ }^{8}$. Dans son Prométhée enchaîné, Eschyle imagine de faire de Prométhée le dépositaire du secret de Thémis, en sorte que Zeus doive un jour composer avec le Titan pour l'heure cloué sur son rocher. On relèvera en particulier cet échange de répliques entre Prométhée et la malheureuse princesse argienne Io, autre célèbre victime de Zeus. À Io qui lui demande :

4 F. BUFFIÈRE, Les mythes d'Homère et la pensée grecque, Paris, $1973^{2}\left[1956^{1}\right.$ ], p. 173-179 (176 pour la citation).

5 G.S. KIRK, The Iliad: a Commentary, Volume I: books 1-4, Cambridge University Press, 1985, p. 95.

6 Laura SLATKIN, The Power of Thetis. Allision and Interpretation in the Iliad, University of California Press, 1991, p. 69-77.

7 C'est là un développement d'une intuition de Gregory NAGY dans les derniers paragraphes de son livre Le meilleur des Acbéens. La fabrique du béros dans la poésie grecque arcbalque, traduit de l'anglais (1979) par Jeannie Carlier et Nicole Loraux, Paris, Seuil, 1994 (Des Travaux), p. 395-396.

8 PIND., VIIL Istbm., 27-40. 
«Est-ce son épouse qui le chassera du trône?», Prométhée répond, tout en gardant l'essentiel de son secret : «En lui enfantant un fils plus fort que son

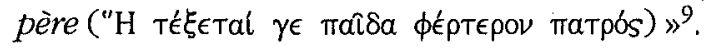

Les expressions parallèles soulignées nous amènent en effet à penser que le père de Briarée doit être Zeus, comme le pensait Félix Buffière à la suite de certains allégoristes. Mais sa mère ne saurait être Thétis puisqu'elle a épousé Pélée et qu'Achille est né. Je pense donc que le mythe du fils éventuel de Thétis et Zeus ne peut pas à lui seul expliquer pleinement notre passage. Il faut, je crois, adjoindre à l'hypothèse de Gregory Nagy et Laura Slatkin une deuxième hypothèse faisant intervenir le mythe parallèle de Métis dans la Théogonie. Mais avant d'argumenter cette thèse, il importe de faire quelques brèves remarques de méthode sur la démarche de ces deux auteurs nordaméricains.

Malgré des différences de détail, leurs travaux sont tout à fait représentatifs de la poétique orale. Or les oralistes refusent en fait d'envisager une intertextualité au sens vrai du terme, c'est-à-dire l'influence d'un texte sur un autre texte. Pour eux les parallélismes, comme ceux que l'on vient de rencontrer dans l'Iliade, la VIIIe Isthmique et le Prométhée enchaîné, renvoient à des traditions poétiques communes dont on doit étudier les manifestations textuelles comme des variantes, sans poser le problème de leur chronologie et de leur genèse éventuelle. Autrement dit la poétique orale est résolument une poétique structurale ou structuraliste.

À cette démarche s'oppose la néo-analyse. Un néo-analyste va se demander si dans tel ou tel cas l'Iliade ne citerait pas une cuwre que nos sources nous permettent d'identifier. Il va donc être amené à se poser des problèmes d'histoire littéraire au sens traditionnel, de datation relative ou absolue des diverses épopées dans le contexte général de la littérature grecque archaïque ${ }^{10}$.

Tout en étant rivales et opposées, ces deux approches devraient pouvoir à l'occasion être combinées de façon fructueuse puisque les phénomènes étudiés présentent probablement des caractères relevant à la fois de l'histoire et de la structure. Dans le cas présent la démarche oraliste est à la fois, on l'a vu, suggestive et insuffisante. Pour introduire une perspective néo-analytique, je ferai d'abord valoir qu'il y a dans l'Iliade deux allusions à une autre variante du mythe de Thétis.

Au chant XVIII, Thétis va demander à Héphaïstos de nouvelles armes pour Achille qui avait prêté les siennes à Patrocle, lequel vient d'être tué et dépouillé de ces armes par Hector. Au dieu forgeron qui s'étonne de sa visite, la déesse marine commence par répondre : «Héphaïstos, est-il une autre des déesses,

9 Esch., Prom., 767-768.

10 Les meilleurs exposés sur la néo-analyse et sa différence avec la poétique orale se trouvent dans W. KULLMANN, Homeriscbe Motive. Beiträge zur Entstebung, Eigenart und Wirkung von Itias und Odyssee, herausgegeben von Roland J. Müller, Stuttgart, Franz Steiner Verlag, 1992. 
habitantes de l'Olympe, dont le coeur ait eu à supporter autant de cruels chagrins que Zeus, fils de Cronos, m'aura octroyé de douleurs, à moi, seule, entre toutes? Seule entre toutes les déesses marines, il m'a soumise à un mortel, Pélée l'Éacide... $»^{11}$. Au chant XXIV, à propos de la différence de valeur entre Hector et Achille, Héra rappelle à Apollon : «Achille est né d'une déesse que j'ai moi-même nourrie et élevée, et donnée comme épouse à un homme, à Pélée, très aimé des immortels... ${ }^{12}$. Selon toute vraisemblance, il s'agit là non pas de deux variantes mythiques à proprement parler mais de deux variations poétiques d'un thème mythique attesté dans l'épopée cyclique des Chants Cypriens et dans le Catalogue des Femmes: Thétis refusa de s'unir à Zeus parce qu'elle avait été élevée par Héra et Zeus, par dépit, décida de la marier à un mortel ${ }^{13}$.

Ce n'est là qu'un cas parmi bien d'autres des fréquentes allusions de l'épopée homérique aux poèmes du Cycle étudiées par des néo-analystes tels que Wolgang Kullmann, dont je viens de citer la publication la plus récente. Pour ma part j'ai récemment été amené à la conclusion que l'Odyssée contenait indubitablement une allusion à une épopée du Cycle aussi tardive que la Télégonie, poème du début du VIe siècle ${ }^{14}$. Je pense que ces phénomènes s'expliquent au mieux dans une optique néo-analytique et néo-wolfienne: l'épopée monumentale écrite n'a été composée sous sa forme finale, après un processus de genèse certes complexe, mystérieux et s'étendant sur de longs siècles, que dans la deuxième moitié du VIe siècle, à une époque où l'usage de l'écriture et les récitations monumentales dans les concours panhelléniques donnent un sens historique clair à la composition monumentale écrite ${ }^{15}$.

Une conséquence particulière et importante de cette thèse historique sur la Question Homérique est que la Théogonie hésiodique doit aussi être antérieure à notre Iliade. Dans un autre article récent, j'ai pu dresser une liste d'une demi-douzaine de parallélismes entre la Théogonie et l'épopée homérique, sétie qui s'explique mieux comme faite de vraies citations que comme de simples parallélismes dus à des traditions communes (thèse oraliste) ${ }^{16}$.

Dans l'optique théologique du présent article, relatif donc au pouvoir de Zeus et à ses relations avec les Olympiens les plus menaçants pour ce pouvoir, il sera instructif de revenir sur l'un de ces parallélismes : l'allusion au Tartare du

11 Iliade, XVIII, 429-433.

12 Iliade, XXIV, 59-61.

13 Chants Cypriens, fr. 2 Bernabé; Catalogue, fr. 210 Merkelbach-West; Pseudo-APOLLODORE, Bibliotbèque, III, 13,5 .

14 A. BAllabriga, La propbétie de Tivésias, in Métis, 4 1989), p. 291-304.

15 À cet égard j'attire l'attention du lecteur sur l'importance du livre encore trop méconnu de Minna Skafte JENSEN, The Homeric Question and the oral-formulaic theory, Copenhagen, Museum Tusculanum Press, 1980.

16 A. BALLABRIGA, La Question Homérique: pour une réouverture du débat, in REG, 103 (1990), p. 16-29. 
chant 8 de l'Iliade. Au début de ce chant, Zeus défend aux dieux d'intervenir sur le champ de bataille de Troie. C'est là une conséquence du courroux d'Achille et de la promesse faite à Thétis. Les paroles de Zeus méritent d'être longuement citées :

Celui que je verrai s'éloigner délibérément des dieux, pour aller porter secours aux Troyens ou aux Danaens, sentira mes coups et s'en reviendra dans l'Olympe en piteux état - à moins que je ne le saisisse et ne le jette au Tartare brumeux, tout au fond de l'abîme qui plonge au plus bas sous terre, où sont les portes de fer et le seutl de bronze, aussi loin au-dessous de l'Hadès que le ciel l'est audessus de la terre. Alors vous comprendrez combien je l'emporte sur tous les

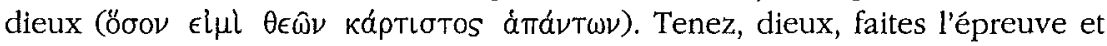
vous saurez, tous. Suspendez donc au ciel un câble d'or; puis accrochez-vous y, tous, dieux et déesses : vous n'amènerez pas du ciel à la terre Zeus, le maître

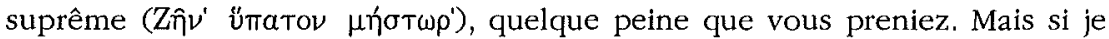
voulais, moi, franchement tirer, c'est la terre et la mer à la fois que je tirerais avec vous. Après quoi, j'attacherais la corde à un pic de l'Olympe, et le tout,

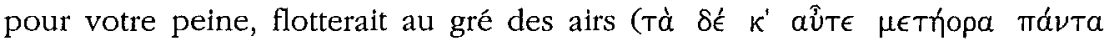
$\gamma \in$ volto). Tant il est vrai que je l'emporte sur les dieux comme sur les hommes ${ }^{17}$.

L'évocation du Tartare aux vers 15-16 (passage souligné) cite deux vers de la Théogonie ( 811 et 720 ). Dans ce poème, la mention d'une porte et d'un seuil d'airain tout au fond de l'univers est motivée du point de vue cosmologique, malgré la difficulté du contexte : il s'agit de la porte et du seuil de la demeure de Nuit, lieu de passage de Nuit et Jour et assise de l'univers opposée au Chaos (Vide) primordial. Or le discours de Zeus aux Olympiens dans le passage cité de l'Iliade fait lui-même allusion au problème de la stabilité cosmique, en des termes («tout flotterait au gré des airs») qui évoquent encore la description du Tartare de la Théogonie: dans le Vide infini qui bée sous le Tartare lui-même tout serait balloté dans un déchaînement de tempêtes (v. 740-744). Mais la perspective est renversée: dans l'Iliade, c'est de l'Olympe et de Zeus que dépend la stabilité cosmique et non d'une terrible et mystérieuse géographie infernale. La porte et le seuil d'airain du Tartare sont ainsi coupés de leur fonction première tandis que le discours de Zeus témoigne d'une réplique spéculative au propos cosmologique de la Théogonie. Avec ce Zeus cosmique, nous sommes plus près du Dieu de Xénophane, Être toutpuissant, transcendant toutes les autres forces du monde, que du dieu combattant de la Théogonie aménageant son jeune pouvoir au-dessus des abîmes du Vide et de la Nuit!

Vers la fin de ce même chant VIII, alors qu'Héra s'indigne de nouvelles menaces proférées par son terrible époux, Zeus fait encore sentir toute son 
autorité à son épouse. Il annonce que les Argiens seront sur la défensive jusqu'à ce que Hector et le Péléide se battent pour le corps de Patrocle et ajoute :

Ainsi en a décidé le destin. De toi, de ta colère, je n'ai nul souci; quand bien même tu t'en irais jusques à ces derniers confins de la terre et de la mer, ou Japet et Cronos sont fixés, privés des doux rayons du soleil d'en haut et des souffles de l'air et n'ayant autour d'eux que le profond Tartare. Non, quand bien même tu t'en irais errer jusque là-bas, de ton dépit je n'ai cure; il n'est pas plus chien que toi ${ }^{18}$.

À leur tour ces vers doivent être rapprochés d'un passage de l'Hymne bomérique à Apollon. Dans une digression sur le mythe de Typhon, terrible "challenger» de Zeus pour la souveraineté céleste, cet hymne conte comment Héra fut amenée à le concevoir dans sa colère contre Zeus qui avait su engendrer dans sa tête la glorieuse Athéné tandis que son propre fils «parthénogénétique » à elle avait été ce boiteux d'Héphaïstos. Pour concevoir ce nouveau fils sans s'unir à Zeus, Héra s'éloigne des dieux en faisant cette invocation :

Écoutez-moi en cet instant, Terre et vaste Ciel de là-haut et vous qui demeurez sous le sol dans le grand Tartare, Dieux Titans dont sont issus les hommes et les dieux ! Entendez-moi, vous tous, en cet instant : donnez-moi un fils, sans Zeus, et qui ne lui soit en rien inférieur pour la force; qu'il l'emporte sur lui au contraire autant que sur Cronos Zeus à la vaste voix ${ }^{19}$.

Ce contact avec les puissances primordiales lui permet d'enfanter le terrible Typhon, le plus sérieux des adversaires de Zeus. Mais toute la mythologie grecque, en dépit de variantes importantes du mythe de Typhon ${ }^{20}$, est au moins unanime sur un point: Zeus l'a finalement emporté sur Typhon. On trouve d'ailleurs une allusion à l'issue de ce combat et au sort de Typhon vers la fin du chant II de l'Iliade, au détour d'une comparaison. Sous les pieds des combattants « le sol gémit comme sous le courroux de Zeus Tonnant quand il cingle la terre autour de Typhée, dans ce pays des Arimes, où l'on dit que gîte Typhée ${ }^{21}$.

Quant à la curieuse vision du chant VIII, celle d'une Héra courroucée, rendant visite aux Titans Iapetos et Cronos à propos d'une péripétie de la guerre de Troie, elle s'expliquerait fort bien comme un écho de l'hymne : les fureurs d'Héra lors de la guerre de Troie resteront sans effet puisqu'aussi bien

\footnotetext{
18 Illade, VIII, $477-483$.

19 Hymne bom. à Apollon, 334-339.

20 J'ai étudié le jeu de ces variantes dans Le demier adversaire de Zeus. Le mythe de Typhon. dans l'épopée grecque arcbaique, in RHR, 207 (1990), p. 3-30.

21 lliade, II, 781-783.
} 
elle a naguère échoué à susciter un rival de Zeus malgré son recours aux puissances primordiales.

Ces quelques exemples peuvent suffire à faire soupçonner la subtilité et la complexité des références théogoniques de l'Iliade. Il ne s'agit pas comme on tend à le penser dans une perspective oraliste de traditions remontant à un passé immémorial mais d'un effort, déjà comparable par exemple à celui d'Eschyle dans ses Prométhées, pour modifier la vision théologique d'œuvres constituées, telles que l'Hymne à Apollon et surtout la Théogonie, et imposer l'idée d'un souverain du ciel en majesté, différent du jeune dieu qui avait dû batailler dur pour s'imposer.

D'ailleurs la Théogonie elle-même, loin de simplement enregistrer les traditions immémoriales du peuple grec, se singularisa fortement dans sa présentation du mythe de Typhon. Alors que les autres traditions archaïques, qui trouveront leur chemin jusque dans la Bibliothèque du Pseudo-Apollodore (IIe siècle ap.), placent la naissance de Typhon sur le tard des temps théogoniques après les naissances d'Héphaïstos et d'Athéna, la Théogonie la place plus haut dans le temps divin avant ces naissances. Cette singularité doit être liée d'une certaine façon à un fait historique important, à savoir que la Théogonie n'est pas plus que l'Iliade un poème primitif de la fin du VIIIe siècle mais une cuvre «deutéro-hésiodique » pour laquelle on peut descendre jusque vers la fin du VIIe siècle ou le début du Ve siècle $a v{ }^{22}$.

Du point de vue théologique, ce déplacement de Typhon en amont du temps divin avait pour but de présenter le règne de Zeus comme d'emblée pacifié. Et pour éliminer définitivement le type de danger qu'avait représenté un Typhon, les aèdes-théologiens de l'«école hésiodique » conçurent l'idée de faire avaler par Zeus la déesse Métis, fille d'Océan et Téthys et incarnation de la prudence et de la ruse :

Et Zeus, le roi des dieux, pour épouse d'abord prit Métis, qui sait plus de choses que tout dieu ou homme mortel. Mais au moment même où elle allait enfanter Athéné, la déesse aux yeux pers, trompant traîtreusement son coeur par des mots caressants, Zeus la mit en sûreté dans ses entrailles, sur les conseils de Terre et de Ciel étoilé. Tous deux l'avaient conseillé de la sorte, pour que l'honneur royal n'appartînt jamais à autre qu'à Zeus parmi les dieux toujours vivants. De Métis en effet le destin voulait que des enfants sortissent sages entre tous - et la vierge aux yeux pers d'abord, Tritogénie, qui de fougue et de sage vouloir a part égale avec son père. Mais elle devait enfanter ensuite un fils au cœur violent qui en̂t été mâ̂tre des hommes et des dieux, si Zeus auparavant

\footnotetext{
22 Voir à ce propos mes articles cités de la $R H R$ (p. 28) et de la $R E G$ (p. 22-23), que développe une analyse plus poussée du proème de la Théogonie: Le Deutéro-Héstode et la consécration de l'bésiodisme, à paraître dans Le métier du mytbe. Hésiode et ses vérités, sous la direction de F. Blaise, P. JUdeT DE la COMBE, Ph. ROUSSEAU, Paris, Ed. du Cerf.
} 


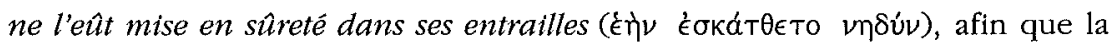
déesse toujours lui fit connaître ce qui lui serait soit heur ou malheur ${ }^{23}$.

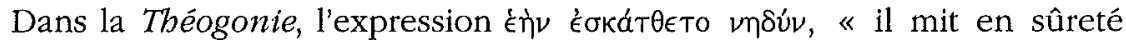

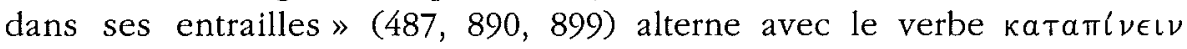
" avaler » (459, 467, 473, 497), tandis qu'un fragment hésiodique racontant l'avalement de Métis par Zeus utilise les deux expressions concurremment à quelques vers de distance ${ }^{24}$. C'est sans doute une façon de signifier que l'«avalement » théogonique n'est pas une dévoration cannibale, comme celle du Cyclope par exemple, puisqu'elle ne détruit pas l'intégrité corporelle des dieux ainsi absorbés et est de ce fait réversible, comme le montre le cas de Cronos régurgitant ses enfants ${ }^{25}$. On doit plutôt considérer qu'un tel avalement est comparable à un emprisonnement ou à des liens, tels ceux qui domptent les dieux vaincus jetés au Tartare. Il n'en reste pas moins que le comportement de Zeus, le roi juste, qui avale une épouse après avoir enchaîné son père, est regrettablement analogue aux agissements de Cronos, le précédent roi des cieux, et faisait donc problème pour une pensée théologique qui s'efforçait de penser l'ordre de Zeus comme plus juste que le règne de Cronos.

Un des plus clairs indices de ces scrupules théologiques se lit dans les Euménides d'Eschyle. À Apollon, qui vient de faire valoir devant un tribunal humain que le meurtre d'Agamemnon, père d'Oreste, par sa femme Clytemnestre pèse plus dans la balance que celui de l'épouse meurtrière par un fils vengeur du père, les Érinyes/Euménides rétorquent, par la voix du Coryphée: «Si l'on t'écoute, Zeus a grand souci du père. Mais lui-même enchaîna $(\not ' \delta \eta \sigma \epsilon)$ son vieux père Cronos. Comment accordes-tu ceci avec cela?». Et Apollon de répondre : "Monstres haïs de la nature entière,

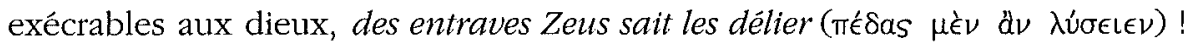
Il y a remède à cela; bien des remèdes existent de s'en dégager. Mais lorsque la poussière a bu le sang d'un homme, s'il est mort, il n'est plus pour lui de résurrection. Mon père contre ce mal n'a point créé de charmes, lui qui bouleverse le monde sans s'essouffler à la peine ! ${ }^{26}$

Effectivement, d'après certaines traditions, Zeus avait finalement fait bénéficier les dieux vaincus, ses anciens adversaires, de sa clémence. Le thêâtre d'Eschyle lui-même illustre remarquablement ces traditions. Au Prométbée

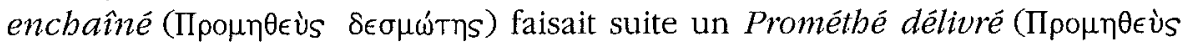
$\lambda \nu \delta \mu \epsilon \nu \circ)$, dont on ne possède plus que quelques fragments, suffisants cependant pour savoir qu'y figurait un chœur de Titans délivrés. De son côté, Pindare,

23 Théogonie, 886-900.

24 Pseudo-HÉs., fr. 343 Merkelbach-West, vers 7 et 10.

25 Voir à ce sujet l'article récent de J. DAVIDSON, Zeus and the stone substitute, in Hermes, 44 (1995), p. 363-369 (364).

26 ESCH., Eum. 640-651. Voir aussi une intéressante variante comique de ce problème dans les Nuées d'ARISTOPHANE (v. 902-909). 


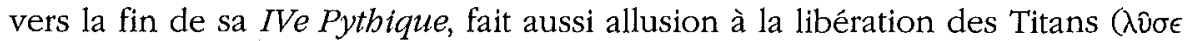

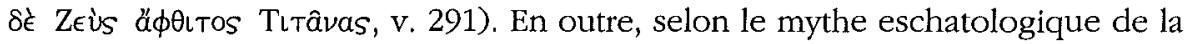
Ile Olympique, Cronos règne sur l'île des Bienheureux, quelque part vers un fond de l'Adriatique situé aux confins septentrionaux de l'univers (v. 77-88). Ce règne de Cronos chez les Bienheureux se retrouve dans une interpolation du mythe des races des Travaux (v. 173a-e).

Ces libérations m'amènent à émettre l'hypothèse que, dans la même optique, la déesse Métis avait dû elle-même bénéficier d'une sortie du corps de Zeus, analogue à celle d'Athéna, sœur de l'éventuel successeur de Zeus, ou encore à celle de Dionysos, cousu dans la cuisse de Zeus avant de renaître comme fils de Zeus ${ }^{27}$. Pour Métis et son fils, comme pour Athéna et Dionysos finalement, la relégation dans le corps de Zeus ne fut pas définitive mais transitoire. Cette sortie du corps de Zeus était sans doute présentée comme un effet de la libre volonté et de la clémence d'un Zeus juste et assuré de son éternel pouvoir et non comme le résultat d'une contrainte analogue à celle par laquelle Cronos fut forcé de régurgiter ses enfants. Ainsi libérés, Métis et son fils pouvaient être des auxiliaires de Zeus pleins de reconnaissance, comme le sont les Cent-Bras dans la Théogonie, liés et relégués au fond de la terre par Ouranos mais ramenés à la lumière et nourris d'ambroisie par Zeus ${ }^{28}$.

Cette analogie avec le sort des Cent-Bras se marque par le fait que le fils de Zeus et de Métis est considéré explicitement comme un Cent-Bras

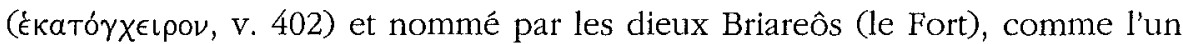
des trois Cent-Bras de la Théogonie, qui est qualifié de «bon » (n’ùv éóvta), exempté de la garde des Titans au Tartare et intégré dans la famille olympienne en devenant gendre de Poséidon ${ }^{29}$. Ce caractère positif de Briarée se retrouve dans une tradition corinthienne rapportée par Pausanias : intervenant à

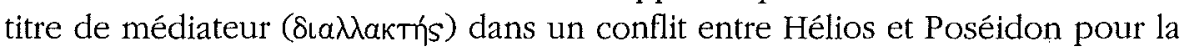
possession de Corinthe, Briarée les départagea en attribuant l'isthme à Poséidon et la hauteur au-dessus de la cité à Hélios ${ }^{30}$. De la sorte, sans pour autant s'identifier à ce Briarée, le fils de Zeus et de Métis apparaît d'une certaine façon comme un doublet et une réplique d'une puissance primordiale terrible mais favorable à l'ordre olympien.

Quant au nom humain de Briarée, Aigaiôn, il semble tiré de la Titanomacbie cyclique, poème sans doute analogue à la Théogonie mais dont il ne subsiste plus qu'une poignée de fragments et témoignages. Dans ce poème, Aigaiôn était le nom d'un fils de Gê et de Pontos qui habitait dans la mer et

27 Les naissances d'Athéna et de Dionysos sont rapprochées par J. DAVIDSON, art. cit. (n. 25), p. 368 n. 27.

28 Théogonie, 617-663.

29 Théogonie, 817-819.

30 PAUS., II, 1, 6. 
combattit aux côtés des Titans ${ }^{31}$. La Titanomachie semble aussi avoir fait mention de «Colonnes d'Aigaiôn » ${ }^{32}$, que d'autres appelaient "Colonnes de Briarée », ancien nom des Colonnes d'Héraclès ${ }^{33}$. Or ces modifications toponymiques présentent un remarquable parallélisme avec le jeu onomastique et théologique de l'Iliade. Alors qu'aux confins occidentaux de l'univers, le nom d'un fils de Zeus s'est substitué à ceux d'Aigaiôn et de Briarée, l'Iliade imagine un fils de Zeus sauveur - comme l'est Héraclès dans la Gigantomachie ${ }^{34}$ portant le double nom d'Aigaiôn et Briarée, le premier nom renvoyant à sa nature de divinité marine, héritée de l'Océanide Métis, le deuxième, plus vrai parce que divin, à sa force susceptible de sauver Zeus.

L'épisode de Briarée sauveur de Zeus est probablement une invention récente de l'Iliade monumentale qui se fonderait, si mon analyse est exacte, sur des spéculations plus larges relatives à la libération de Métis et motivées par un sentiment de piété, d'eusebeia, tendant à attribuer à Zeus un comportement plus digne que celui de Cronos.

Il reste maintenant à revenir sur le type de relation qui peut exister entre Briarée, le fils de Zeus «supérieur à son père », et la déesse Thétis. Au départ il existe des affinités significatives entre Thétis, fille du Vieux de la Mer Nérée, et Métis, fille d'Océan. Il s'agit en effet de deux divinités marines, objets du désir de Zeus et douées d'un pouvoir de métamorphose par lequel elles tentent d'échapper à leur prétendant, Zeus ou Pélée ${ }^{35}$. En outre l'avalement de Métis ne suffisait pas à régler aux yeux de tous le problème de la perennité du pouvoir de Zeus. J'ai rappelé un peu plus haut que la Théogonie antidatait, si j'ose dire, le combat de Zeus contre Typhon de façon à présenter l'avalement de Métis comme une solution définitive. Mais la chronologie des autres traditions continuait à faire problème puisque, à la suite de la naissance d'Athéna, intervenaient non seulement le combat contre Typhon mais aussi le combat des dieux contre les Géants, que la Théogonie préfère passer sous silence.

J'avancerais donc encore l'hypothèse que cette double donnée - affinité de Métis et Thétis, insuffisance de l'avalement de Métis - conduisit à la création de la variante du mythe de Thétis attestée dans Pindare et Eschyle. Autrement dit le thème du danger représenté par un fils de Thétis et Zeus serait un

31 Titanomacbie, fr. 3 Bernabé. C'est par inadvertance que G. NAGY, op. laud. (n. 7), p. 396, écrit qu'Aigaiôn combattit contre les Titans (against the Titans); le texte dit bien toís Titãoı $\sigma v \mu \mu a \chi \epsilon \tilde{\imath} \nu$.

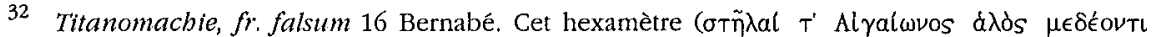
$\Gamma(\gamma a v t o s)$ offre un texte corrompu mais on peut, je crois, retenir la notion de "Colonnes d'Aigaiôn".

33 ÉLIEN, Histoire Variée, V, 3, qui cite Aristote.

34 Voir les allusions à ce rôle d'Héraclès dans la Théogonie, 954 et le Bouclier, 28-29.

35 Ps. APOLl., Bibl., I, 3, 6 (Métis); III, 13, 5 (Thétis). Voir à ce propos l'analyse de J.-P. VERNANT dans Les ruses de l'intelligence. La mètis des Grecs, Paris, 1974, p. 104-124 (109). 
doublet secondaire du mythe de Métis et Zeus ${ }^{36}$. Il évite l'avalement d'une déesse par Zeus - impie et inutile - et se situe chronologiquement vers la fin de l'âge des héros. La menace - cette fois réellement ultime - sur le règne de Zeus, après celles représentées par l'éventuel fils de Métis, Typhon, les Géants, est conjurée de la façon la plus élégante qui soit, en mettant la déesse interdite dans les bras d'un mortel.

Ainsi Thétis sera bien la seule à souffrir de cette mésalliance. Tous les autres, dieux et hommes, s'en réjouissent; les dieux parce qu'ils échappent à un ultime péril et les hommes que cette ultime alliance honore, avant la séparation représentée par la guerre de Troie, où le fils de 'Thétis et Pélée va s'illustrer plus que tout autre. À cet égard l'union de Thétis et Pélée est tout à fait parallèle à celle d'Aphrodite et d'Anchise. De façon significative ces deux dernières unions entre déesses et mortels se suivent à la fin de la Théogonie (10061010). Elles préparent la fin de l'âge des héros, caractérisé par un mélange de l'humain et du divin qui doit s'achever avec la guerre de 'Troie ${ }^{37}$.

Cette séparation des hommes et des dieux a pour corollaire un pouvoir de Zeus sur lequel en principe ne doit plus planer aucun péril. Mais si malgré tout il s'en présentait un, postérieur à l'avalement, suivi de libération, de Métis et au mariage de Thétis avec un mortel ? Telle me semble être la question qui a donné naissance à l'épisode de Thétis et Briarée au chant I de l'Iliade. Pour résumer ma thèse, un rhapsode, un Homéride selon moi, imagina une Thétis allant chercher, à l'occasion d'une querelle sur l'Olympe, le fils de Métis, qui est aussi le fils que Thétis n'a pas pu avoir. Ce fils de Métis, on songea à lui donner un double nom humain et divin qui renvoyait à la fois au caractère anti-olympien d'Égéon dans la Titanomacbie cyclique et au caractère olympien du CentBras Briarée dans la Théogonie hésiodique. Et ce sauveur de Zeus doit habiter sous les eaux des confins cosmiques, comme le suggère le fait que l'expression «Colonnes de Briarée » soit un ancien nom des "Colonnes d'Héraclès». Cette invention de l'Iliade est ainsi une sorte d'ultime surenchère et radicalisation par rapport à toutes les spéculations théologiques dont j'ai tenté de donner une idée.

Mais nous avons vu aussi que l'Iliade faisait à l'occasion allusion à l'ancien mythe de Thétis, celui des Chants Cypriens et du Catalogue des Femmes, qui ne faisait pas encore état d'un fils dangereux pour Zeus mais simplement du souci de Thétis de ne pas déplaire à Héra. Cette complexité référentielle de l'Iliade annonce dans une certaine mesure la combinaison des deux variantes

36 Je ne pense pas qu'il ait intérêt, à propos de la figure de Thétis, de tenir compte des spéculations sur une Thétis puissance primordiale suscitées par la découverte sur un papyrus, publié en 1957, d'un commentaire physique et allégorique à un poème d'Alcman. À mon sens en effet Gl.W. MOST [ Alcman's "cosmogonic" fragment (fr. 5 Page, 81 Calame), in CQ, 37 (1987), p. 1-19] a donné de sérieuses raisons de penser que nous avons simplement affaire à une lecture allégorique analogue à celles que j'ai rappelées à propos du mythe de Thétis et Briarée - d'un partbeneion, dans lequel Alcman devait conter le mythe de la poursuite de Thétis par Pélée.

37 Voir dans ce même numéro mon étude du mythe d'Énée. 
du mythe de Thétis dans les Argonautiques d'Apollonios de Rhodes : Thétis refuse d'abord de céder au désir de Zeus par égard pour Héra qui l'a élevée; mais Zeus ne renonce pas; il continue d'épier la Néréide jusqu'à ce que Thémis révèle que le destin de Thétis est d'enfanter un fils meilleur que son père

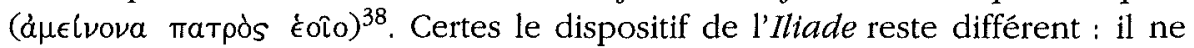
s'agit pas de combiner subtilement les deux variantes du mythe de Thétis en un même passage mais de les utiliser dans des passages éloignés du poème (chants I, 18 et 24 ).

Il n'en reste pas moins qu'il n'y a pas entre la poétique béllénistique et la poétique archaique l'abîme que l'on postule couramment. Il y a certes entre les deux toute une série de différences, bien décrites dans tout bon manuel de littérature grecque. Mais ces différences, qui font généralement l'objet essentiel de tout travail historique en tant que tel, ne nous autorisent jamais à passer d'un relativisme historique normal à un relativisme intégral, qui postule en l'occurrence une radicale hétérogénéité des poétiques anciennes. Par réaction contre cette tendance hyperrelativiste en histoire grecque, j'attire l'attention sur une certaine continuité des poétiques, qui s'explique d'ailleurs historiquement fort bien pour peu que l'on admette que nos poèmes épiques sont des textes évolués du VIe siècle et non des «sagas primitives » du VIIIe siècle

Dans cette optique, soucieuse donc de repérer différences et continuités historiques, on doit même descendre jusqu'au dispositif d'écriture d'un mythographe tel que le Pseudo-Apollodore, dont la Bibliothèque (IIe siècle ap.) demeure d'ailleurs un outil irremplaçable pour l'analyse de la mythologie grecque, ce qui mérite chaque fois un grand coup de chapeau. À son tour spécifique et différent des écricures poétiques archaïques ou hellénistiques, le résumé tardif en prose énumère simplement les variantes du mythe de Thétis (Selon certains... D'autres disent que...) ${ }^{39}$. Mais les choix qui s'ouvrent toujours à une prose d'époque romaine ne font que reconduire pour l'essentiel ceux qui s'offraient déjà aux poètes vers la fin de l'archaïsme, à l'issue d'une période (VIIe-VIe siècles) d'intense activité intellectuelle qui avait beaucoup enrichi la mythologie " primitive » des IXe-VIIIe siècles.

Et c'est dans ce cadre historique général que nous devons replacer les rapports entre l'Iliade et la Théogonie. D'une part les poèmes homériques sont beaucoup plus récents qu'on ne l'enseigne d'ordinaire : leurs auteurs sont en mesure, dans la deuxième moitié du VIe siècle, de jouer, de façon déjà savante et raffinée, à partir d'une riche production épique elle-même proche de son état classique. D'autre part, au sein de cette production épique, la Théogonie,

38 APOLL. RHOD., Arg., IV, 790-809. La formule soulignée est sans doute une réminiscence de

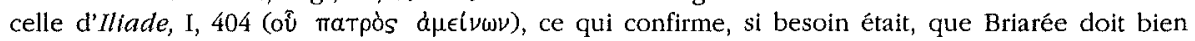
être un fils de Zeus.

39 Ps. APOLL., Bibl. III, 13, 5. On notera que la variante récente est exposée en premier et l'ancienne en second. C'est sans doute dû au fait que la variante récente avait été consacrée par les auteurs classiques (Pindare, Eschyle) tandis que l'ancienne se trouvait dans des textes vieillis et peu lus (Chants Cypriens, Catalogue). 
tout au long du Vle siècle, fut une œuvre incontournable par rapport à laquelle se situèrent aussi bien des poètes épiques que des théologiens réformateurs tels que Phérécyde de Syros, Épiménide de Crète voire Xénophane de Colophon. À cet égard la Théogonie joua au Vle siècle un rôle analogue mutatis mutandis à celui du poème philosophique Sur la Nature de Parménide d'Élée dans l'histoire de la pensée du Ve siècle ${ }^{40}$.

Centre d'Anthropologie

Alain BALLABRIGA (CNRS, EHESS)

56, rue du Taur

$\mathrm{F}-31000$ TOULOUSE

40 La présente étude est issue d'une conférence faite lors de la Septième rencontre du Groupe de contact interuniversitaire pour l'étude de la religion grecque antique (F.N.R.S.) à l'Université Catholique de Louvain ( 8 février 1995). Je remercie vivement mes collègues belges de leur invitation, de la riche discussion qui suivit mon intervention et, enfin, de l'accueil fait à ce texte dans la revue Kernos. 